\title{
ACESSIBILIDADE PARA PESSOAS IDOSAS: DESAFIO DO ENVELHECIMENTO POPULACIONAL NO BRASIL
}

\section{ARTIGO DE REVISÃO}

BARBOSA, Renata Viana ${ }^{1}$, FERREIRA, Crisley Mara de Azevedo ${ }^{2}$

BARBOSA, Renata Viana. FERREIRA, Crisley Mara de Azevedo. Acessibilidade para pessoas idosas: desafio do envelhecimento populacional no Brasil. Revista Científica Multidisciplinar Núcleo do Conhecimento. Ano 06, Ed. 06, Vol. 12, pp. 93104. Junho de 2021. ISSN: 2448-0959, Link de acesso: https://www.nucleodoconhecimento.com.br/saude/pessoas-idosas, 10.32749/nucleodoconhecimento.com.br/saude/pessoas-idosas

\section{RESUMO}

A Organização Mundial de Saúde (OMS) define como idoso o indivíduo com 60 anos ou mais. Essa parcela da população tem crescido em um ritmo mais rápido que os demais grupos etários. A transição demográfica tem implicações severas em importantes esferas da vida social, como o mercado de trabalho, a previdência e as necessidades de saúde. Desse modo, o envelhecimento desafia a produzir políticas de saúde responsivas às demandas das pessoas idosas e que estimulem a capacidade funcional do indivíduo no cotidiano da sociedade. $\mathrm{O}$ artigo trata-se de uma abordagem qualitativa de caráter exploratório por meio de revisão bibliográfica, com o objetivo de levantar as ações propostas voltadas para o envelhecimento de qualidade no Brasil, identificando as iniciativas feitas para atender a demanda da população

\footnotetext{
${ }^{1}$ Bacharelada Em Enfermagem.

2 Orientadora. Mestrado em Química.
}

RC: 89175

Disponível em: https://www.nucleodoconhecimento.com.br/saude/pessoas-idosas 
idosa, delimitando-a e analisando a efetividade das medidas em vigor destinada a esse público.

Palavras-chave: Envelhecimento populacional, Políticas públicas, Acessibilidade para idosos, Direitos dos idosos, Linha de cuidado.

\section{INTRODUÇÃO}

A Organização Mundial da Saúde (WORLD HEALTH ORGANIZATION, 2015), define como idoso o indivíduo com idade igual ou superior a 60 anos. Essa idade mínima de classificação pode variar de acordo com as condições de desenvolvimento de cada país, para efeito de formulação de políticas públicas. É importante ressaltar que o envelhecimento não está apenas ligado a idade cronológica, existem variações no que diz respeito as condições de saúde, grau de participação na sociedade, nível de dependência ou independência entre os idosos, em diversas situações.

A parcela da população acima de 60 anos tem crescido em um ritmo mais rápido que os demais grupos etários em todo o mundo. Atualmente o Brasil conta com mais de 28 milhões de pessoas nessa faixa etária, número que representa $13 \%$ da população do país, o IBGE divulgou em 2018 uma projeção de que esse percentual tende a dobrar nas próximas décadas. O esperado para 2050 é que o percentual da população mundial acima de 60 anos ultrapasse o percentual de crianças, o que nunca ocorreu historicamente (BNDES,2017).

O Envelhecimento populacional tem estreita ligação com os processos de transição demográfica e epidemiológica. A demográfica no que diz respeito às variações dos níveis de natalidade e de mortalidade além dos movimentos migratórios, o que colaborou para modificar a participação dos três grupos etários, tornando a população mais envelhecida ou jovem (OLIVEIRA, 2019), o que tem implicações severas em importantes esferas da vida social, como o mercado de trabalho, a previdência e as necessidades de saúde. Em relação aos fatores epidemiológicos podemos citar:

RC: 89175

Disponível em: https://www.nucleodoconhecimento.com.br/saude/pessoas-idosas 
urbanização, hábitos alimentares, sedentarismo, acidentes de trânsito, violência urbana e cobertura de saneamento (REIS; BARBOSA; PIMENTEL, 2016). A interação entre a transição demográfica e a epidemiológica, são impactantes na saúde, uma vez que o envelhecimento populacional implica aumento da prevalência de causas de morbidade e mortalidade associadas a doenças crônicas e degenerativas. Sendo assim, ao se analisar o processo de envelhecimento da população brasileira como um todo, verifica-se relevância do país se estruturar para quando for, de fato, um país de idosos.

Alterações na estrutura etária da sociedade trazem oportunidades e desafios que devem ser equacionados no devido tempo para que não se percam as chances de extrair proveito das oportunidades trazidas pelo bônus demográfico e para que os desafios não se tornem graves problemas sociais e econômicos (CARVALHO, 2019). Cita-se como um fator desencadeante desses desafios, a ausência de políticas públicas com ofertas reais e efetivas de prevenção e cuidado, subsidiando a organização de uma rede destinada a infraestrutura adequada as necessidades do público idoso. A Política Nacional do Idoso (PNI), Política Nacional de Saúde da Pessoa Idosa (PNSPI) e o Estatuto do Idoso são instrumentos importantes para embasar ações voltadas a qualidade de vida do idoso, garantindo seus direitos e demarcando sua cidadania. Porém, para a efetivação de política pública é imprescindível iniciativas éticas, cidadãs e morais por parte de todos os envolvidos, permitindo a reeducação da população diante dos reconhecimentos dos desafios enfrentados por esse grupo etário (MARTINS et al., 2007).

Ainda que já existam essas leis, é notório suas falhas, uma vez que, baseando no direito do idoso a mobilidade (incluída como prioridade no Estatuto do Idoso através do projeto de lei $n$. 7061, de 2017) era para ser dever do estado garantir a estrutura adequada para a livre circulação de idosos usuários de transporte público, devendo haver remoção de barreiras e obstáculos que impeçam ou dificultem a movimentação, o que acaba não ocorrendo.

RC: 89175

Disponível em: https://www.nucleodoconhecimento.com.br/saude/pessoas-idosas 
Desse modo, o envelhecimento desafia a produzir políticas de saúde responsivas às demandas das pessoas idosas e que estimulem a capacidade funcional do indivíduo no cotidiano da sociedade (VERAS et al., 2018). No que diz respeito a Saúde, destacase a importância de melhorias relacionadas ao acesso, ao cuidado integral, a criação e efetividade de ações intersetoriais de acordo com a especificidade e a demanda. Cabe destacar que a pessoa idosa é mais vulnerável a eventos adversos e apresentam características mais delicadas quanto a condução dos agravos em saúde, necessitando intervenções mais direcionadas e multissetoriais com foco no cuidado (BRASIL, 2019).

O presente estudo tem como objetivo levantar as ações propostas voltadas para o envelhecimento de qualidade no Brasil, identificando as iniciativas feitas para atender a demanda da população idosa, delimitando-a e analisando a efetividade das medidas em vigor destinada a esse público.

\section{METODOLOGIA}

A fim de desenvolver a problemática em questão, realizou-se uma pesquisa qualitativa, de caráter exploratório por meio de revisão bibliográfica. Nesse sentido, para a seleção das fontes, foram consideradas adequados seguindo critérios de inclusão, bibliografias que abordassem os desafios do público-alvo, medidas adotadas ao longo dos anos para minimizar esses desafios e as políticas públicas que destacaram o direito dos idosos. Como critérios de exclusão foram utilizados: artigos que não abordavam sobre o contexto relacionado ao tema e que estavam fora do recorte temporal, teses, anais de congressos ou conferência, livros, relatórios técnicos ou científicos totalizando 15 estudos excluídos.

Assim, com o intuito de atender aos objetivos propostos por este trabalho, cabe destacar que foram eleitos 25 artigos retirados do Scientific Electronic Library Online (Scielo) Ministério da saúde e Google Acadêmico através da análise dos dados de 2014 a 2020. A coleta e a interpretação dos dados seguiram as seguintes etapas: a) RC: 89175

Disponível em: https://www.nucleodoconhecimento.com.br/saude/pessoas-idosas 
busca estabelecendo palavras chaves como: envelhecimento populacional, políticas públicas, acessibilidade para idosos, direitos dos idosos; linha de cuidado; b) leitura exploratória de todo o material selecionado; c) delimitação dos fragmentos principais para aprofundamento das questões relacionadas ao conteúdo temático.

\section{RESULTADOS E DISCUSSÃO}

A Organização das Nações Unidas (ONU) difere o conceito de idoso entre países desenvolvidos e subdesenvolvidos. Nos primeiros, são consideradas idosas as pessoas com 65 anos ou mais, enquanto nos países subdesenvolvidos, essa denominação é atribuída aos indivíduos com 60 anos ou mais.

Para Veras et al. (2018) um grande feito da humanidade foi a ampliar o tempo de vida, que se deu acompanhado pela melhora dos parâmetros de saúde da população. Embora essa melhoria não seja homogênea e distribuída de forma equitativa em todos os países, chegar à velhice, que antes era privilégio de poucos, passa a ser a norma, mesmo nos países em desenvolvimento. Esta conquista maior do século XX transformou-se, no entanto, no grande desafio para o século atual.

Conforme Miranda; Mendes e Silva (2016) o crescimento da população idosa é um fenômeno mundial, e, no Brasil, as modificações se dão de forma radical e bastante acelerada. As projeções mais conservadoras já indicavam que, em 2020 seremos o sexto país do mundo em número de idosos com um contingente superior a 30 milhões de pessoas, chegando ao ano de 2050 como quinto maior país do planeta em número de habitantes com 253 milhões de pessoas, ficando abaixo apenas da Índia, China, EUA e Indonésia. Conforme censo demográfico 2020 divulgado pelo Instituto Brasileiro de Geografia e Estatística (IBGE), o Brasil tem mais de 28 milhões de pessoas na faixa etária a partir de 60 anos, representando 13\% da população do país.

Para Reis et al., (2017), no Brasil essa transição deve ocorrer já em 2030, conforme mostra o Gráfico 1. Segundo esses autores, o envelhecimento pode ser explicado não

RC: 89175

Disponível em: https://www.nucleodoconhecimento.com.br/saude/pessoas-idosas 
somente pelos avanços ocorridos nos indicadores em saúde, mas também, pode ser esclarecido por dois fatores-chave: o aumento da expectativa de vida e a queda da taxa de fecundidade. Segundo o Banco Nacional de Desenvolvimento BNDES, (2017) nos últimos anos houve uma elevação da expectativa de vida ao nascer em todo o mundo. Em 1950, a expectativa de vida era de 46,8 anos, em 2015, esse passou para 70,4 , e espera-se que em 2030 chegue a 74,5 anos.

Conforme estudo publicado pela World Health Organization (2015), outro fator relevante da transição demográfica atual é a longevidade, boa parte dessa população que irá viver muitos anos. Entre 2015 e 2030, é esperado que o grupo de idosos acima de 85 anos aumentará em um ritmo superior que a população abaixo de sessenta anos, podendo alcançar 7\% do total de pessoas em 2030, como demonstrado no Gráfico 2 (BNDES, 2017). A maior probabilidade de morte ocorre, cada vez mais, em idades mais avançadas, calcula-se, por volta de $2030,78 \%$ das mortes no Brasil ocorrerão com pessoas acima de sessenta anos. Vale destacar que, em 1950, apenas $18 \%$ das pessoas chegavam a idades superiores a 60 anos (WORLD HEALTH ORGANIZATION, 2015).

$\mathrm{RC}: 89175$

Disponível em: https://www.nucleodoconhecimento.com.br/saude/pessoas-idosas 
Gráfico 1 - Proporção da população com idade até 14 anos e acima de sessenta anos, 1980-2070

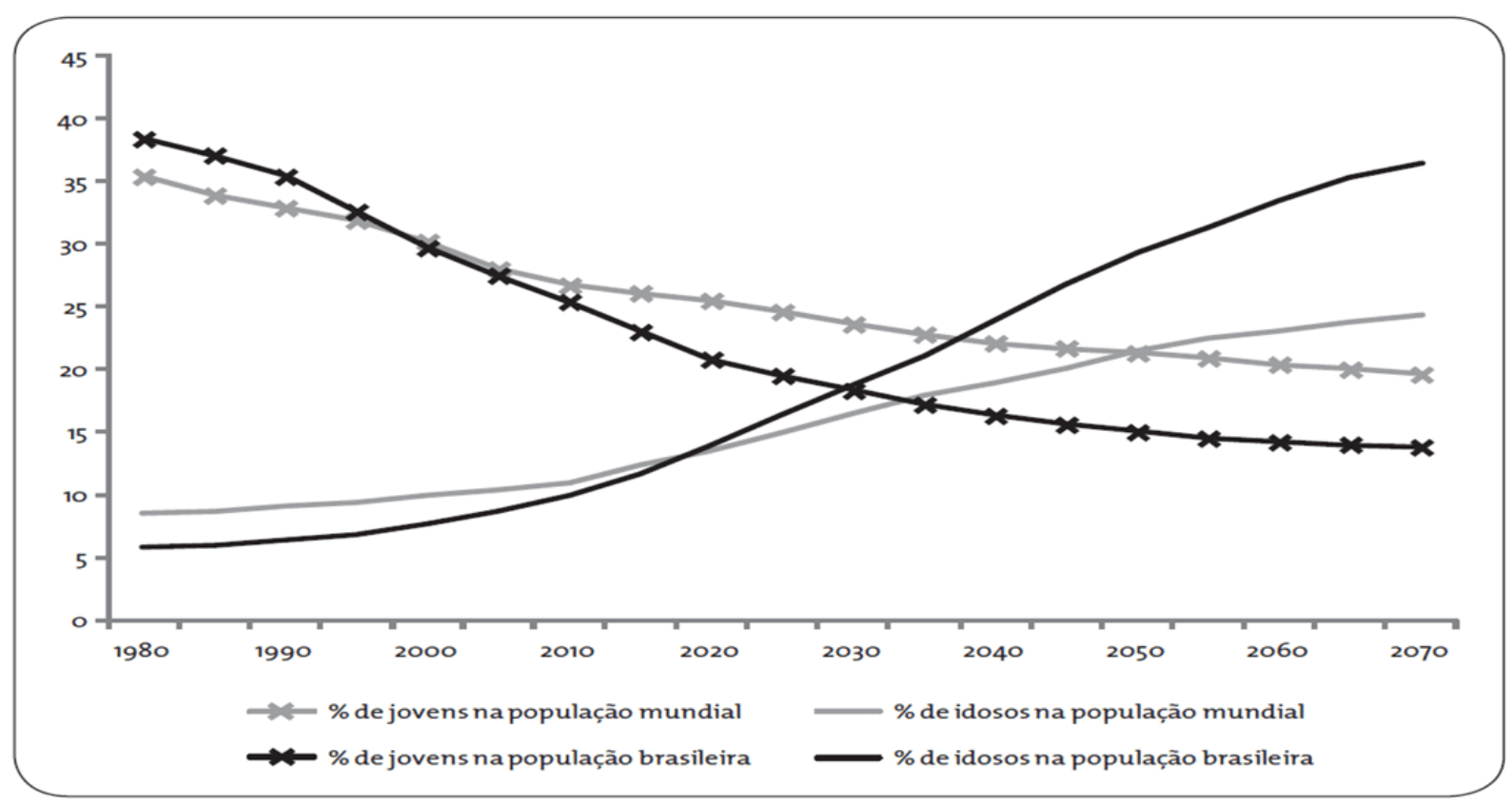

Fonte: REIS; BARBOSA; PIMENTEL, 2016.

RC: 89175

Disponível em: https://www.nucleodoconhecimento.com.br/saude/pessoas-idosas 
Gráfico 2 - Taxa de crescimento populacional por faixa etária: mundo e Brasil, 20152030

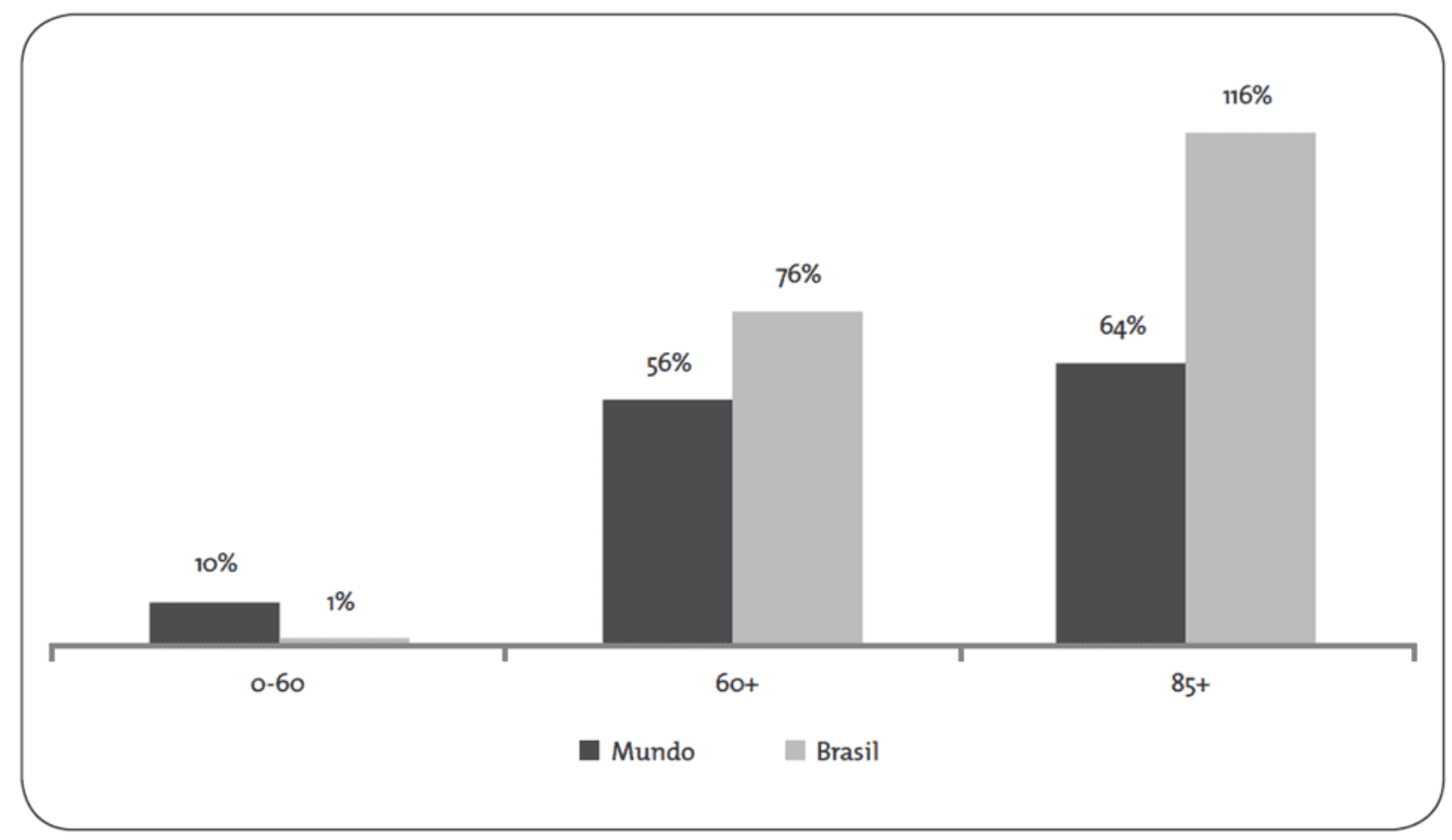

Fonte: REIS; BARBOSA; PIMENTEL, 2016.

Diante desse cenário é explícito a importância do país em estruturar-se, já que apesar de a expectativa de vida aumentar, a qualidade de vida por sua vez, não acompanha esse avanço.

O envelhecimento é dito por Bastos, Almeida e Fernandes (2017) como um processo fisiológico, dinâmico e progressivo. Desta forma, torna-se pressentível, possibilitando aos países oportunidades de planejarem-se e adaptarem-se as demandas dessa população cada dia mais crescente. Pinto e Neri (2013) descreve que o Brasil não está preparado para lidar com está realidade que está posta, uma vez que não atender as necessidades dessa população de maneira efetiva e com qualidade. As políticas públicas garantidas pela Constituição e pelo Estatuto do Idoso não são trabalhadas

RC: 89175

Disponível em: https://www.nucleodoconhecimento.com.br/saude/pessoas-idosas 
adequadamente, os idosos na grande maioria sentem-se mais excluídos do que acolhidos por esses programas, que acabam sendo fragmentados e focalizados.

Assim sendo, torna-se indispensável exigir dos governos e população, meios de garantir a proteção e efetivação dos direitos da pessoa idosa, previsto em lei de forma concreta, é necessário também promover meios de divulgar os documentos legais que regulamentam as políticas públicas direcionadas à promoção do bem-estar, garantia de respeito e dignidade aos idosos brasileiros (FREEDOM, 2017).

A população em foco, conta com dispositivos legais que garantem seus direitos e preservam sua autonomia, integração e participação efetiva na sociedade como instrumento de cidadania. A primeira política que regulamenta direitos específicos para esse segmento foi resultado de encontros e debates que foram realizados por meio de vários seminários que culminaram no documento intitulado "Políticas para a Terceira Idade nos anos 90", que originou mais tarde, em 1994, na Política Nacional do Idoso - Lei no 8.842, de 04 de janeiro de 1994. A partir dessa política, várias outras foram sendo instituídas, com destaque para o Estatuto do Idoso - Lei ㄲo 10.741, de 03 de outubro de 2003 e Política Nacional de Saúde da Pessoa Idosa, aprovada pela Portaria no 2.528 de 03 de outubro de 2006. Contudo é evidente suas lacunas no que diz respeito a eficiência (ESCOBAR; MOURA, 2016).

Damasceno e Sousa (2016) salienta que de acordo com o Ministério Público, uma deficiência da Política Nacional do Idoso é a falta de especificação da lei que puna pessoas que utilizem de preconceito contra o idoso. Sendo o envelhecimento um processo natural da população, não deveria existir discriminação de nenhuma natureza no que diz respeito a esse processo, Costa et al. (2020) acentua que o estatuto do idoso foi criado com a finalidade de solucionar os problemas associados a população idosa, tendo dificuldade em seu desempenho devido ao mau costume social. Quando entrou em vigor tinha resultados esperados de sua eficácia, porém não resolveu o problema, apresentando-se impotente para o fim a qual fora sancionada,

RC: 89175

Disponível em: https://www.nucleodoconhecimento.com.br/saude/pessoas-idosas 
necessitando de um grau superior de eficácia. Afirma também que, a adversidade geradora dessa má eficiência não se encontra na legislação, mas sim, na maneira em que ela é empregada.

Assim, Pinheiro, Areosa (2018), declara que as políticas ainda são frágeis e o sistema público de saúde não está totalmente adequado e preparado para atender de forma plenamente satisfatória a população idosa e sua família. Dessa forma, indaga que é necessário aperfeiçoar as políticas públicas e legislações vigentes, para que haja interação das responsabilidades entre o estado, sociedade e família em relação a demanda dessa população.

Embora ainda apresente lapsos, as políticas públicas também foram percussoras de inovações relacionado ao envelhecer saudável no Brasil. Muniz (2017) descreve algumas das conquistas feitas enfatizando, o atendimento preferencial no Sistema Único de Saúde (SUS), reserva de 10\% dos assentos dos transportes públicos coletivos concedendo o benefício da gratuidade ou desconto de $50 \%$ no valor das passagens, programas de atividades físicas e em saúde, desconto de $50 \%$ sobre o valor de ingressos para eventos artísticos e culturais, preferência em filas e atendimentos no setores público e privado.

No entanto, no que diz respeito ao custo de vida, o jornal Estado Minas em maio de 2015 reportou que a vida na terceira idade torna-se mais cara, deixando claro que;

\footnotetext{
Enquanto a população em geral sofre com o aumento dos preços, para essa parcela o aperto tem sido mais intenso e é o maior dos últimos 12 anos. Para eles, que precisam de um bom plano de saúde, de alimentação adequada e, em muitos casos, de uma quantidade significativa de remédios, o dragão não perdoa e está cada vez mais feroz. (VIDIGAL, 2015).
}

Dessa maneira, com o avançar da idade, as funções orgânicas decorrentes do processo de envelhecimento, em consequência dos agravos ao longo da vida, passam a ser mais vulneráveis ao declínio funcional, com consequente redução de

RC: 89175

Disponível em: https://www.nucleodoconhecimento.com.br/saude/pessoas-idosas 
capacidades e atividades, incluindo dois tipos de desvantagens: perdas físicas, relacionadas à diminuição do vigor, da agilidade, da resistência e da força muscular, se comparadas a outras etapas da vida; e perda de saúde, definida como maior ocorrência de problemas de saúde, com suas implicações e maior dificuldade de recuperação (AMTHAUER; FALK, 2014).

Correlacionado as perdas físicas, é garantido o direito do idoso de ser assegurado viver de forma plena, com condições adequadas que garantam saúde, lazer, segurança e locomoção. Os caminhos para transformar esse anseio em realidade são muitos: perpassam desde a instituição e efetividade de políticas públicas sólidas, a implementação de infraestrutura urbanística planejada e acessível e ao uso de tecnologias assistivas de forma homogênea e integrativas, bem como a busca por alternativas para adaptar melhor ao que já foi implantado (FREEDOM, 2017).

\section{CONSIDERAÇÕES FINAIS}

O envelhecimento está acontecendo rapidamente, porém as tecnologias voltadas para a qualidade no acolhimento dessa população não acompanham o mesmo ritmo. À vista disso, nos artigos selecionados, foi permitido compreender que o maior desafio está na implementação de políticas públicas efetivas, voltadas para a comodidade desse público e a devida atenção a esse avanço do processo de envelhecer no Brasil.

Neste caso, cabe as autoridades governamentais atentarem-se para execução dos direitos nos quais a lei promove, uma vez que, o envelhecimento é previsível por tratarse de uma fase fisiológica em que todo o ser humano passará. O Brasil dispõe desde 2003, de uma das leis mais avançadas relativa as garantidas estabelecidas para os idosos, correspondendo ao estatuto do idoso, que por sua vez, ficou claramente evidenciado no decorrer da pesquisa, sua aplicação ineficiente, bem como, de outras legislações correspondentes.

RC: 89175

Disponível em: https://www.nucleodoconhecimento.com.br/saude/pessoas-idosas 
Referente a acessibilidade, tanto no âmbito de saúde, quanto no âmbito de mobilidade, torna-se necessário implantação de condições favoráveis. Novas estruturas urbanísticas e de saúde deverão ser desempenhadas para atender as necessidades do público em questão.

Consequentemente, é inegável a constatação de que o Brasil não está preparado ou preparando-se para essa transição demográfica. Os idosos têm que lidar com as limitações naturais da idade e sobretudo, com a imaturidade da mentalidade social.

\section{REFERÊNCIAS}

AMTHAUER, Camila; FALK, João. Percepção de profissionais da saúde acerca das dificuldades enfrentadas pelos idosos. Idosos, Revista de Enfermagem UFPE online. Recife, v. 8, n. 11, nov. 2014. Disponível em: https://periodicos.ufpe.br/revistas/revistaenfermagem/article/download/13615/16450. Acesso em: 9 maio 2020.

BASTOS, Rosângela Alves Almeida; ALMEIDA, Francisca das Chagas Alves de; FERNANDES, Maria das Graças Melo. Adaptação psicossocial de idosos em tratamento hemodialítico: uma análise à luz do Modelo de Roy. Revista Enfermagem UERJ. Rio de Janeiro, v. 25, n. 1, p. 1-6, 31, ago. 2017. Disponível em: http://fiadmin.bvsalud.org/document/view/ycqyr. Acesso em: 6 maio 2020.

BRASIL. Ministério da Saúde. Saúde da pessoa idosa: prevenção e promoção à saúde integral. Brasília, DF: Ministério da Saúde, 16 set. 2019. Disponível em: https://saude.gov.br/saude-de-a-z/saude-da-pessoa-idosa. Acesso em: 2 jun. 2020.

CARVALHO, Dilma de Maria. Os desafios de envelhecer no Brasil. Portal do envelhecimento e longeviver. São Paulo, 16 jan. 2019, p. 4-6. Disponível em: https://www.portaldoenvelhecimento.com.br/os-desafios-de-envelhecer-no-brasil/. Acesso em: 9 maio 2020.

RC: 89175

Disponível em: https://www.nucleodoconhecimento.com.br/saude/pessoas-idosas 
COSTA, Amanda et al. A eficácia do estatuto do idoso nos aspectos penais. In: JORNADA JURÍDICA, 10., 2020. Anápolis. Anais [...]. Anápolis: UniEvangelica. 2020. Disponível em:

JORNADA JURÍDICA, 10. http://repositorio.aee.edu.br/bitstream/aee/8853/1/paper\%202\%20PERIODO\%20LU CIANO\%20DO\%20VALE.pdf. Acesso em: 8 maio 2020.

DAMASCENO, Carolinne Kilcia Carvalho Sena; SOUSA, Cristina Maria Miranda de. Análise sobre as políticas públicas de atenção ao idoso no Brasil. Revista Interdisciplinar, Teresina, v. 1, n. 9, p. 185-190, set. 2016. Disponível em: https://dialnet.unirioja.es/descarga/articulo/6772023.pdf. Acesso em: 7 maio 2020.

ESCOBAR, Karin Alves do Amaral; MÔURA, Flávia Aparecida de. Análise de políticas sociais para idosos no Brasil: um estudo bibliográfico. Cadernos Unifoa, Volta Redonda, v. 1, n. 30, p. 47-55, abr. 2016. Disponível em: http://revistas.unifoa.edu.br/index.php/cadernos/article/view/376/435\#: :text=A\%20pr imeira\%20pol\%C3\%ADtica\%20que\%20regulamenta,na\%20Pol\%C3\%ADtica\%20Na cional\%20do\%20ldoso\%20\%2D. Acesso em: 6 maio 2020.

FREEDOM. Direito do idoso: a mobilidade na terceira idade. [S. I.], 2017. p. 4-6. Atualizado em 13 jul. 2020. Disponível em: https://blog.freedom.ind.br/direito-doidoso-a-mobilidade-na-terceira-idade/. Acesso em: 9 maio 2020.

MARTINS, Josiane de Jesus; SCHIER, Jordelina, ERDMANN, Alcoque Lorenzini; ALBUQUERQUE, Gelson Luiz de. Políticas públicas de atenção à saúde do idoso: reflexão acerca da capacitação dos profissionais da saúde para o cuidado com o idoso. Revista Brasileira de Geriatria e Gerontologia, Rio de Janeiro, v. 10, n. 3, p. 371-382, set./dez. 2007.

Disponível em:

http://www.scielo.br/scielo.php?script=sci_arttext\&pid=\$1809-

98232007000300371\&lng=en\&nrm=iso. Acesso em: 10 ago. 2020.

RC: 89175

Disponível em: https://www.nucleodoconhecimento.com.br/saude/pessoas-idosas 
MIRANDA, Gabriella Morais Duarte; MENDES, Antonio da Cruz Gouveia; SILVA, Ana Lucia Andrade da. $O$ envelhecimento populacional brasileiro: desafios e consequências sociais atuais e futuras. Revista Brasileira de Geriatria e Gerontologia, Rio de Janeiro, v. 19, n. 3, p. 507-519, maio/jun. 2016. Disponível em: https://www.scielo.br/scielo.php?pid=S1809-

98232016000300507\&script=sci_arttext\&tlng=pt. Acesso em: 5 maio 2021.

MUNIZ, Raquel. Terceira idade: conquistas asseguradas. Hoje em Dia, Belo Horizonte, 31 jul. 2017.2 Disponível em: https://www.hojeemdia.com.br/opini\%C3\%A3o/colunas/raquel-muniz1.456804/terceira-idade-conquistas-asseguradas-1.547287. Acesso em: 8 maio 2020.

OLIVEIRA, Anderson Silva. Transição demográfica, transição epidemiológica e envelhecimento populacional no Brasil. Hygeia - Revista Brasileira de Geografia Médica e da Saúde, Uberlândia, v. 15, n. 32, p. 69-79, jun. 2019. Disponível em: http://www.seer.ufu.br/index.php/hygeia/article/view/48614. Acesso em: 5 maio 2020.

PINHEIRO, Osvaldo Daniel dos; AREOSA, Silvia Virginia Coutinho. A importância de políticas públicas para idosos. Revista Brasileira de Assuntos Regionais e Urbanos, Goiânia, v. 4, n. 2018. $\quad$ 2, Disponível em: http://seer.pucgoias.edu.br/index.php/baru/article/view/6724. Acesso em: 20 maio 2020

PINTO, Juliana Martins, NERI, Anita Liberalesso. Doenças crônicas, capacidade funcional, envolvimento social e satisfação em idosos comunitários: estudo fibra. Ciência \& Saúde Coletiva, Rio de Janeiro, v. 18, n. 12, p. 3449-3460, 2013. Disponível em: https://www.scielosp.org/article/csc/2013.v18n12/3449-3460/pt/. Acesso em: 9 maio 2020.

REIS, Carla; BARBOSA, Larissa Maria de Lima Horta; PIMENTEL, Vitor Paiva. O desafio do envelhecimento populacional na perspectiva sistêmica da saúde. BNDES

RC: 89175

Disponível em: https://www.nucleodoconhecimento.com.br/saude/pessoas-idosas 
Setorial, Rio de Janeiro, n. 44, p. [87] -124, set. 2016. Disponível em: https://web.bndes.gov.br/bib/jspui/handle/1408/9955. Acesso em: 10 maio 2020.

VERAS, Renato et al. Envelhecer no Brasil: a construção de um modelo de cuidado. Ciência \& Saúde Coletiva, Rio de Janeiro, v. 23, n. 6, jun. 2018. Disponível em: https://doi.org/10.1590/1413-81232018236.04722018. Acesso em: 2 jun. 2020.

VIDIGAL, Luciane. Alta do custo de vida consume renda dos idosos. Estado de Minas, Belo Horizonte, 4 maio 2015. Economia. Disponível em: https://www.em.com.br/app/noticia/economia/2015/05/04/internas_economia,643622 /dragao-queima-ainda-mais-a-renda-dos-idosos.shtml. Acesso em: 10 maio. 2020.

WORLD HEALTH ORGANIZATION. Active ageing: a policy framework. Geneva, 2015. $59 \quad$ p. Disponível em: https://web.archive.org/web/20150319062526/http://whqlibdoc.who.int/hq/2002/who_ nmh_nph_02.8.pdf. Acesso em: 5 maio 2020.

Enviado: Setembro, 2020.

Aprovado: Junho, 2021.

RC: 89175

Disponível em: https://www.nucleodoconhecimento.com.br/saude/pessoas-idosas 